\title{
Foreign-body conjunctival granuloma secondary to finger-nail trauma
}

\author{
V. Sowmya, Nelly E.P. Nazareth, Vijna B. Kamath \\ Department of Ophthalmology, Fr Muller Medical College, Kankanady, Mangalore, \\ Karnataka, India
}

\begin{abstract}
A foreign body lodged in the conjunctiva can trigger a granuloma formation. In the past, cilia, caterpillar hair, insect wing and fibers have been reported to incite foreignbody granuloma formation in the eye. Trauma to the eye with finger nail is frequently encountered but goes unnoticed among the pediatric population. Finger-nail trauma to the eye leading to a mass has not been reported in literature. We hereby report a case of conjunctival foreign-body granuloma in a seven-year-old child following finger-nail trauma. Excision biopsy and histopathology of the mass affirmed the diagnosis.
\end{abstract}

Key words: Conjunctival granuloma, foreign body, finger nail

\section{Introduction}

Granuloma formation can occur in the conjunctiva as an acute or chronic inflammatory response to foreign materials lodged in it. We herein report a rare case of foreign-body granuloma secondary to lodgement of a finger-nail chip within the bulbar conjunctiva in a seven-year-old boy following a trauma with his brother's finger nail. To the best of our knowledge, this is the first report in literature of a foreign-body granuloma formation in response to a finger nail.

\section{Case}

A seven-year-old boy was brought in by his parents after noticing redness, swelling of the lids and a mass in the left eye for four days following a trivial trauma with his brother's finger nail while playing ten days before. On examination, his bestcorrected visual acuity was $20 / 20$ in both eyes. Slit-lamp examination revealed nasal conjunctival congestion, conjunctival mass measuring $10 \times 10 \mathrm{~mm}$ on the nasal side about $5 \mathrm{~mm}$ away from the limbus near the insertion of medial rectus muscle. The mass was pink in color with a smooth surface and well-defined margins (Fig. 1). The rest of the ocular examination was unremarkable.

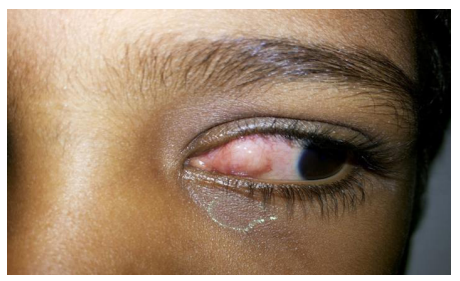

Fig. 1. Left conjunctival mass and conjunctival congestion.

Correspondence: Dr V. Sowmya, MBBS, DOMS, DNB, Department of Ophthalmology, Fr Muller Medical College, Kankanady, Mangalore, Karnataka, India - 575002 
Base-line blood investigations were normal. The ultrasound report showed a conjunctival mass with variable intralesional echogenicity suggesting inflammatory pathology (Fig. 2). MRI of the orbit showed altered signal intensity in the medial extraconal space of the left orbit which was mildly hyperintense on short $\mathrm{TI}$ inversion recovery (STIR) with mild contrast enhancement adjacent to medial rectus insertion (Fig. 3).

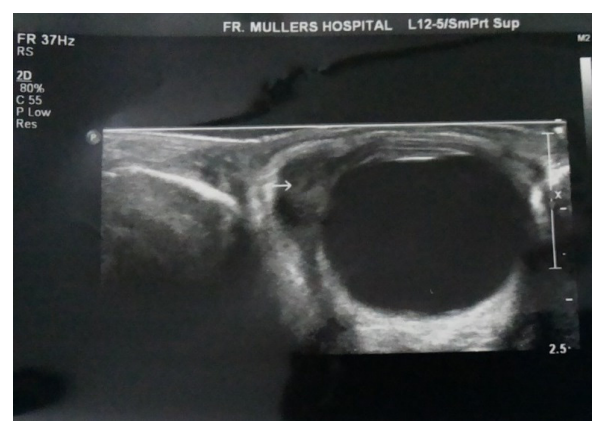

Fig. 2. Ultrasound of the left eye showing the conjuctival mass with variable echogenicity.

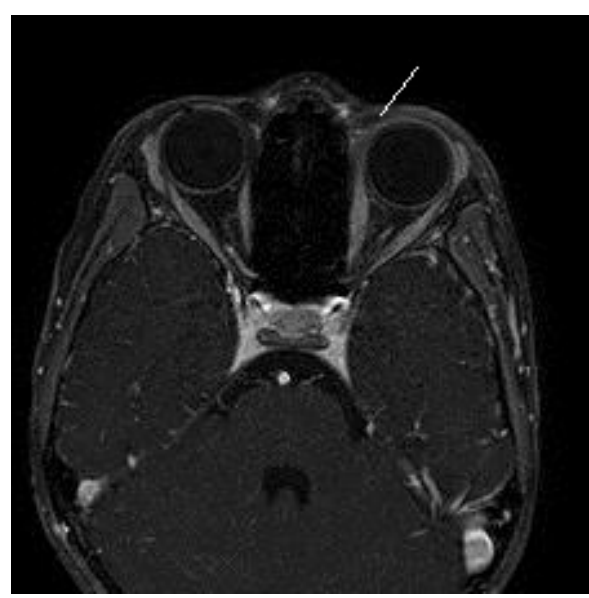

Fig. 3. MRI orbit showing left conjunctival mass.

Conjunctival abscess, pyogenic granuloma and foreign-body granuloma were contemplated as differential diagnoses. The patient was started on systemic and topical antibiotics along with systemic anti-inflammatory drugs. No change in the mass was observed even after five days of treatment. The patient underwent excision biopsy of the conjunctival mass under general anesthesia (Figs. 4 and 5). Histopathology showed fibrocollagenous tissue with keratin flakes surrounded by foreign-body giant cell reaction along with inflammatory infiltrate composed of eosinophils, lymphocytes, plasma cells and histiocytes [Figs. 6 and 7). Periodic acid-Schiff (PAS) stain of the given tissue was negative for fungus and Ziehl Neelsen Stain was negative for Mycobacterium tuberculosis arriving upon a diagnosis of foreign-body granuloma. The postoperative period was uneventful with the child receiving tapering dose of antibiotic-steroid drops for four weeks following the excision biopsy (Fig. 8). No recurrence of the lesion has been observed during the last six months of follow-up.

\section{Discussion}

Foreign-body granuloma can be acute or chronic in onset. It can develop rapidly and may go undiagnosed at times. ${ }^{1}$ When a foreign body gets lodged in the conjunctiva, initially there is an acute inflammatory response in the form of exudation of plasma and fibrin dislodging it. However, when the foreign body has a large 


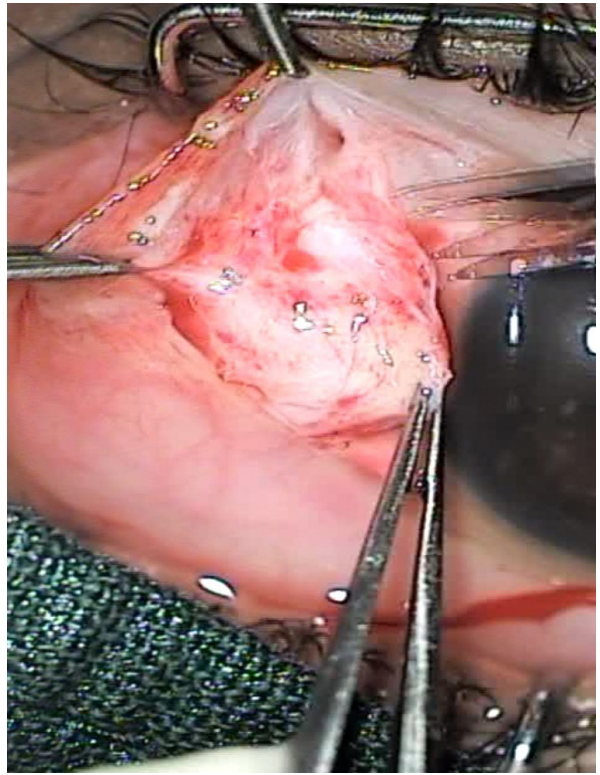

Fig. 4. Excision of the conjunctival mass.

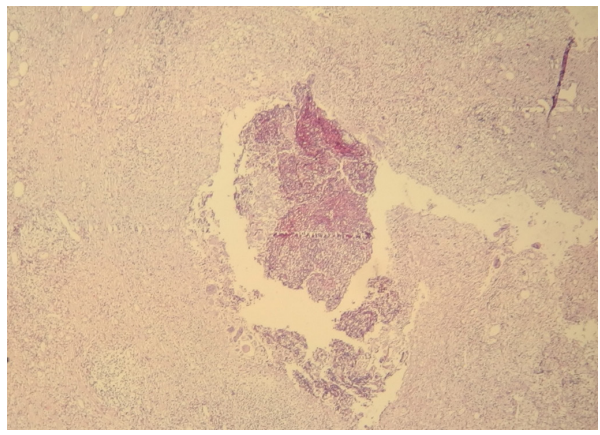

Fig. 6. Photomicrograph showing a central keratin debris with surrounding inflammation ( $H \& E, 40 X)$.

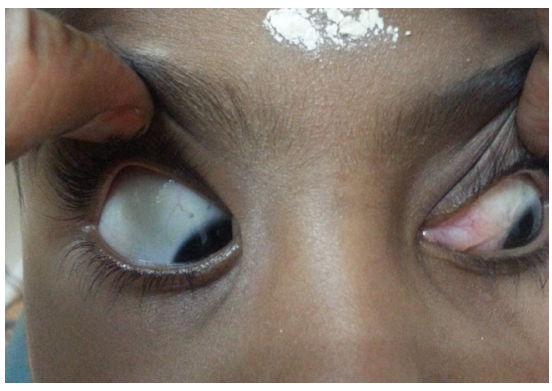

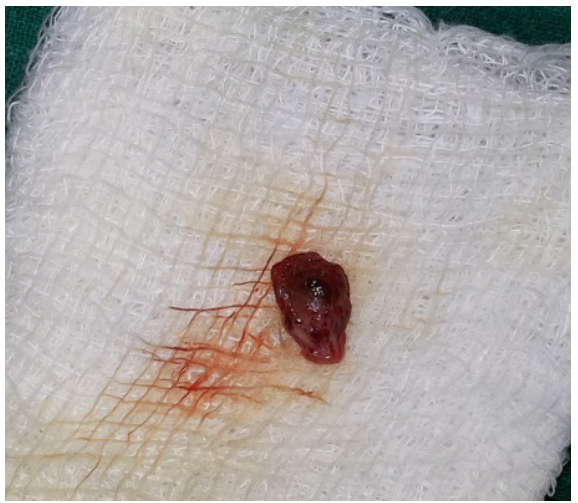

Fig. 5. Post-excision gross specimen.

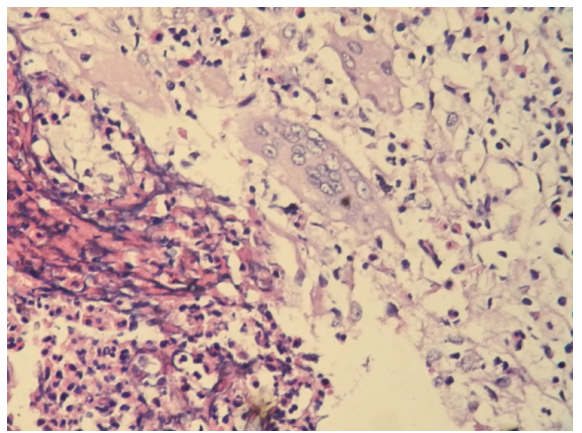

Fig. 7. Photomicrograph showing keratin debris with a foreign body giant cell, plasma cell and histiocytes (H \& E, $400 \mathrm{X}$ ).
Fig. 8. One week post-operative picture showing quiet eye with full extra-ocular movements. 
surface area, this mechanism may be insufficient and the foreign body becomes embedded. ${ }^{2,4}$ This is followed by a chronic inflammatory response resulting in the formation of a foreign body granuloma. ${ }^{2,4}$

Agents reported in literature inciting granuloma formation in the conjunctiva include cilia, caterpillar hair, ${ }^{3}$ insect wing ${ }^{4,5}$ and natural and synthetic fibers (teddy bear granuloma). ${ }^{6}$ However, there are no reports of foreign-body conjunctival granuloma associated with finger nails (Medline search). This case is reported to emphasize that granuloma formation in the conjunctiva can occur following finger-nail trauma in children. Various studies have shown that nail dust is rich in endotoxins and able to stimulate large-scale release of IL-8, a potent neutrophil-attracting chemokine which would cause a chronic inflammation through well-documented cell-stimulatory effects of endotoxin. ${ }^{7,8}$ Keratin in finger nails can trigger the protective mechanisms of the body for removal of a foreign substance and give rise to foreign-body granuloma mimicking conjunctival abscess. The excision of the lesion and histological study provide diagnostic certainty and healing.

\section{Conclusion}

Recognition of foreign-body conjunctival granuloma is a challenge to an ophthalmologist. Finger-nail granuloma could be common, but under-reported. Finger-nail trauma is very common in children, which should be kept in mind while evaluating children with conjunctival mass.

\section{References}

1. Roy FH, Hanna C. Foreign body granuloma of the conjunctiva. Ann Ophthalmol 1978;10:1361-1362.

2. Weinberg JC, Eagle RC, Jr, Font RL, Streeten BW, Hidayat A, Morris DA. Conjunctival synthetic fiber granuloma: a lesion that resembles conjunctivitis nodosa. Ophthalmology 1984;91:867-872.

3. Watson PO, Sevel D. Ophthalmianodosa. Br J Ophthalmol 1966;50:209-217.

4. Venkatesh $P$, Lakshmaiah NC, Chawla R. Insect wing conjunctival granuloma. Cornea 2003;22:489-490.

5. Babu K, Maralihalli RE. Insect wing tarsal foreign body causing conjunctival granuloma and marginal keratitis. Indian J Ophthalmol 2009;57(6):473-474.

6. Ferry AP. Synthetic Fiber Granuloma ('Teddy Bear' Granuloma) of the Conjunctiva. Arch Ophthalmol 1994;112(10):1339-1341.

7. Millar NA, Burrow J G, Hay J, Stevenson R, Putative risks of ocular infection for chiropodists and podiatrists. Brit J Podriat Med 1996:51(11):158-160.

8. Donaldson C, Carline T, Brown D, Gilmour P, Donaldson K and ELRGInColt Laboratories, Edinburgh University. Brit J Podiatry 2003;6(1):28-32. 